12 months; NCT01877668]), or had an IR to $\geq 1$ TNFi (OPAL Beyond [n=394; 6 months; NCT01882439]). Pts were randomised to receive tofacitinib $5 \mathrm{mg}$ twice daily (BID), tofacitinib $10 \mathrm{mg} \mathrm{BID}$, adalimumab $40 \mathrm{mg}$ subcutaneous injection once every 2 weeks (OPAL Broaden only) or PBO (advancing to tofacitinib 5 or $10 \mathrm{mg}$ BID at Month 3, OPAL Broaden and OPAL Beyond), in addition to continuing on a single, stable csDMARD. Composite endpoints assessed: Psoriatic Arthritis Disease Activity Score (PASDAS), Disease Activity Score using 28 joints with C-reactive protein, Disease Activity Index for Reactive Arthritis/Psoriatic Arthritis (DAREA/DAPSA) and Composite Psoriatic Disease Activity Index (CPDAI).

Results: Demographics and baseline disease characteristics were generally similar between treatment groups within the 2 studies, except for duration of PsA disease (longer in OPAL Beyond) and geographic distribution (OPAL Broaden having more Eastern EU pts). Baseline values for composite endpoints were generally similar across treatment groups and studies (table 1). Both doses of tofacitinib showed improvements in composite endpoints vs PBO at Month 3 in both studies (table 1). In OPAL Broaden, the effects of adalimumab were similar to both doses of tofacitinib across composite endpoints. Effect size for the composite endpoints (using a subpopulation of pts who had all available data for all endpoints) was highest for PASDAS and typically lowest for DAREA/DAPSA; this rank order of effect size was similar across treatment arms and studies. At Month 3 , effect sizes in pts receiving active treatment ranged from 0.90 (DAREA/DAPSA for tofacitinib $5 \mathrm{mg} \mathrm{BID)}$ to 2.40 (PASDAS for tofacitinib $10 \mathrm{mg}$ BID) in OPAL Broaden, and 0.81 (DAREA/DAPSA for tofacitinib $5 \mathrm{mg} \mathrm{BID)}$ to 1.84 (PASDAS for tofacitinib $10 \mathrm{mg} \mathrm{BID)}$ ) in OPAL Beyond (table 1). Standardised response means generally followed the same pattern as effect size across studies with both doses of tofacitinib (table 1).

Abstract THU0323 - Table 1. Summary of mean at baseline, LS mean change from baseline, effect size and standardised response mean at Month 3 for composite endpoints (PASDAS, DAS28-3(CRP), DAREA/DAPSA, CPDAI) in OPAL Broaden and OPAL Beyond studies

\begin{tabular}{|c|c|c|c|c|c|c|c|}
\hline & \multicolumn{4}{|c|}{ UPAL Broaden } & \multicolumn{3}{|c|}{ OPAl Beygad } \\
\hline & $\begin{array}{c}\text { Tnfaritininih } \\
5 \mathrm{mi} \text { BIID } \\
\text { N-107 }\end{array}$ & $\begin{array}{c}\text { Tofaritinit } \\
10 \mathrm{mg} \text { BII } \\
\mathbf{N}-104\end{array}$ & 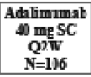 & $\begin{array}{l}\text { PAaraln } \\
\mathbb{N}=105\end{array}$ & 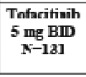 & $\begin{array}{c}\text { Tnfuritimilh } \\
10 \mathrm{ng} \text { BID } \\
\text { N-1x2 }\end{array}$ & $\begin{array}{l}\text { PAarehth } \\
N=131\end{array}$ \\
\hline \multicolumn{8}{|c|}{ Beseline meaul (SD) [N1] } \\
\hline PASDAS & $\begin{array}{l}6.03 \\
(1.15)\end{array}$ & $\begin{array}{c}6.01 \\
(1.60)\end{array}$ & $\begin{array}{l}5.92 \\
(1.25)\end{array}$ & $\begin{array}{l}5.03 \\
1.1 .19\end{array}$ & $\begin{array}{l}5.09 \\
(1.22)\end{array}$ & $\begin{array}{l}6.43 \\
(-21) \\
-219\end{array}$ & $\begin{array}{l}597 \\
(126)\end{array}$ \\
\hline & & & [106] & [103] & [124] & {$[128]$} & {$[128]$} \\
\hline DAS28-3(CRP) & $\begin{array}{l}4.56 \\
(0.92)\end{array}$ & $\begin{array}{l}4.48 \\
0.577\end{array}$ & $\begin{array}{l}4.38 \\
(1.02)\end{array}$ & $\begin{array}{l}4.50 \\
(1.097)\end{array}$ & $\begin{array}{l}4.51 \\
(1.04)\end{array}$ & $\begin{array}{l}4.67 \\
(-177\end{array}$ & $\begin{array}{l}440 \\
(103)\end{array}$ \\
\hline & [107] & {$[104]$} & {$[106]$} & {$[105]$} & {$[131]$} & [132] & [131] \\
\hline DAREADAPSA & $\begin{array}{l}45.55 \\
0.033) \\
{[107]}\end{array}$ & $\begin{array}{c}43.59 \\
0.519 \\
{[104]}\end{array}$ & $\begin{array}{c}38.52 \\
(18.177 \\
{[105]}\end{array}$ & $\begin{array}{l}43.8 \\
02.40 \\
{[105]}\end{array}$ & 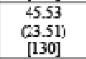 & $\begin{array}{r}51.54 \\
027.803 \\
{[132]}\end{array}$ & $\begin{array}{c}42.64 \\
(22.09) \\
{[131]}\end{array}$ \\
\hline CPDAI" & $\begin{array}{c}9.9 \\
(2.39) \\
{[81]}\end{array}$ & $\begin{array}{l}100 \\
{[2.76)} \\
{[00]}\end{array}$ & $\begin{array}{c}9.7 \\
(2.87) \\
{[77]}\end{array}$ & $\begin{array}{c}9.9 \\
{[2.69)} \\
{[8.1]}\end{array}$ & $\begin{array}{c}10.1 \\
(2.58) \\
{[79]}\end{array}$ & $\begin{array}{c}10.7 \\
(2.59) \\
{[79]}\end{array}$ & $\begin{array}{c}9.6 \\
(286) \\
{[35]}\end{array}$ \\
\hline \multicolumn{8}{|c|}{ LS mean change from baseline at Mouth 3 (SE) [N2] } \\
\hline PASDAS & $\begin{array}{c}-1.9900 * * \\
(0.14) \\
{[104]}\end{array}$ & $\begin{array}{c}-2.39 * 0 * \\
(0.14) \\
{[102]}\end{array}$ & $\begin{array}{c}-2.17^{\mathrm{k}+0 *} \\
(0.14) \\
{[106]}\end{array}$ & $\begin{array}{l}-1.21 \\
0.19 \\
{[101]}\end{array}$ & $\begin{array}{c}-1.93 \cdots 0 \\
0.14) \\
{[121]}\end{array}$ & $\begin{array}{c}-2.144^{* a 1} \\
(0.14) \\
{[124]}\end{array}$ & $\begin{array}{l}-0.83 \\
(014) \\
{[126]}\end{array}$ \\
\hline DAS28-3(CRP) & $\begin{array}{c}-1.33^{* * * * *} \\
0.100 \\
{[107]}\end{array}$ & $\begin{array}{c}-1.63^{* * * *} \\
0.100 \\
{[104]}\end{array}$ & $\begin{array}{c}-1.51 \mathrm{lnos} \\
0.100 \\
{[106]}\end{array}$ & $\begin{array}{l}-0.77 \\
0.11) \\
{[104]}\end{array}$ & $\begin{array}{c}-1.38^{* 40} \\
0.100 \\
{[130]}\end{array}$ & $\begin{array}{c}-1.23^{301} \\
(1.10) \\
{[132]}\end{array}$ & $\begin{array}{l}-0.61 \\
0101 \\
{[131]}\end{array}$ \\
\hline DAREADAPSA & 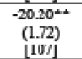 & $\begin{array}{c}-24.40^{0+1+4} \\
(1 . j 3) \\
{[104]}\end{array}$ & $\begin{array}{l}-19.10^{\circ} \\
(1.77) \\
103] \\
103\end{array}$ & $\begin{array}{l}-13.79 \\
1.827 \\
11041\end{array}$ & $\begin{array}{c}-22.46^{+14} \\
(1.67) \\
{[129]}\end{array}$ & $\begin{array}{c}-21.04 \\
(. .70) \\
{[152\rfloor}\end{array}$ & $\begin{array}{l}-1.60 \\
(169) \\
{[131]}\end{array}$ \\
\hline CPDAI" & $\begin{array}{c}-2.9 \\
(0.34) \\
{[91]}\end{array}$ & $\begin{array}{c}-4.2^{300} \\
(0.36) \\
{[6]}\end{array}$ & $\begin{array}{c}-3.1^{*} \\
(0.34) \\
{[77]}\end{array}$ & $\begin{array}{l}-2.2 \\
0.30) \\
{[00]}\end{array}$ & $\begin{array}{c}-9.3^{m *} \\
0.31) \\
{[78]}\end{array}$ & $\begin{array}{c}-3.4 * * \\
(0.311) \\
{[70]}\end{array}$ & $\begin{array}{c}-1.6 \\
(0.61) \\
{[13]}\end{array}$ \\
\hline \multicolumn{8}{|l|}{ Effect size at Month 3 } \\
\hline & $\mathrm{N} 3=68 \mathrm{~B}$ & $\mathrm{~N} 3=62$ & $\mathrm{~N} 3=96$ & $\mathrm{~N} 3=77$ & $k 3=64$ & $\mathrm{~N} 3=62$ & $\mathrm{~N}=72$ \\
\hline PASTDAS & 173 & 240 & 16 & 101 & 153 & 184 & 074 \\
\hline DAS2R-3(CRP) & 1.47 & 1.57 & 1.37 & 0.73 & 1.07 & L.16 & 063 \\
\hline DAYEAVDAYSA & 0.90 & 1.25 & $1.0 \mathrm{~s}$ & 0.50 & 0.81 & 0.84 & 051 \\
\hline CDMAT & $1 \mathrm{nz}$ & 147 & 105 & 060 & 141 & 44 & 050 \\
\hline \multicolumn{8}{|c|}{ Standardised response mean at Menth 3} \\
\hline PASDAS & 1.42 & 1.75 & 1.73 & 1.19 & 1.20 & .53 & 005 \\
\hline DAS2E-3(CRP) & 1.25 & 1.46 & $1.5)$ & 0.79 & 1.14 & .29 & 061 \\
\hline DAREADAPSA & 1.05 & 1.25 & 1.47 & 0.78 & 2.94 & 4.15 & 038 \\
\hline CPDAI & 0.89 & 1.27 & 1.11 & 0.80 & 1.11 & L.49 & 063 \\
\hline 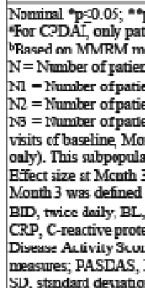 & 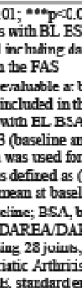 & 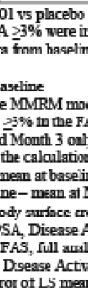 & 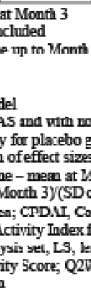 & 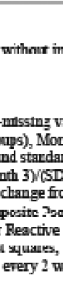 & 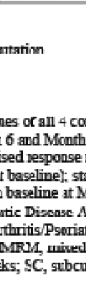 & 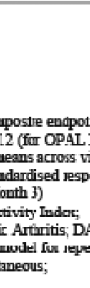 & $\begin{array}{l}\text { Is accoss } \\
\text { roaden } \\
\text { its } \\
\text { ase mean at } \\
\text { ise-3, }\end{array}$ \\
\hline
\end{tabular}

Conclusions: In 2 Phase 3 studies, tofacitinib $5 \mathrm{mg}$ and $10 \mathrm{mg}$ BID improved composite endpoint scores vs PBO over 3 months in pts with PsA. The largest effect size and standardised response means were observed for PASDAS. Effect sizes and standardised response means varied across endpoints but were consistent across studies.
Acknowledgements: Study sponsored by Pfizer Inc. Medical writing support was provided by $C$ Viegelmann of $C M C$ and funded by Pfizer Inc.

Disclosure of Interest: P. Helliwell Grant/research support from: AbbVie, Jans sen, Pfizer Inc, Consultant for: AbbVie, Janssen, UCB, Speakers bureau: AbbVie, Amgen, Janssen, Pfizer Inc, L. Coates Grant/research support from: AbbVie Janssen, Consultant for: AbbVie, Amgen, BMS, Celgene, Eli Lilly, Janssen, MSD Novartis, Pfizer Inc, Sun Pharma, UCB, O. FitzGerald Grant/research suppor from: AbbVie, BMS, Novartis, Pfizer Inc, Consultant for: Amgen, Celgene, Eli Lilly, Janssen, P. Nash Grant/research support from: AbbVie, BMS, Eli Lilly, Janssen, Novartis, Pfizer Inc, Roche, Sanofi, UCB, Consultant for: AbbVie, BMS, Eli Lilly, Janssen, Novartis, Pfizer Inc, Roche, Sanofi, UCB, Speakers bureau: AbbVie, BMS, Eli Lilly, Janssen, Novartis, Pfizer Inc, Roche, Sanofi, UCB, E. Soriano Grant/research support from: AbbVie, Janssen, Novartis, Pfizer Inc, UCB, Consultant for: AbbVie, Janssen, Novartis, Pfizer Inc, UCB, Speakers bureau: AbbVie, BMS, Janssen, Novartis, Pfizer Inc, Roche, UCB, M. E. Husni Consultant for: Abb Vie, Amgen, BMS, Eli Lilly, Janssen, Novartis, Pfizer Inc, Regeneron, UCB, M.-A Hsu Shareholder of: Pfizer Inc, Employee of: Pfizer Inc, K. Kanik Shareholder of: Pfizer Inc, Employee of: Pfizer Inc, T. Hendrikx Shareholder of: Pfizer Inc, Employee of: Pfizer Inc, J. Wu Shareholder of: Pfizer Inc, Employee of: Pfizer Inc E. Kudlacz Shareholder of: Pfizer Inc, Employee of: Pfizer Inc DOI: 10.1136/annrheumdis-2018-eular.1275

\section{THU0324 DISEASE ACTIVITY AND PATIENT CHARACTERISTICS BY COMORBIDITY AMONG PSORIATIC ARTHRITIS (PSA) PATIENTS IN A US REGISTRY}

${ }^{1}$ P.J. Mease, H.J. Litman ${ }^{2}$, N.A. Accortt ${ }^{3}$, S. Rebello ${ }^{2}$, J.D. Greenberg ${ }^{4}$, H. Feng ${ }^{2}$ M.M. Gharaibeh ${ }^{3}$, G.A. Aras ${ }^{3}$, D.H. Collier ${ }^{3} .{ }^{1}$ Swedish Medical Center and University of Washington, Seattle WA; ${ }^{2}$ Corrona LLC, Waltham MA; ${ }^{3}$ Amgen Inc., Thousand Oaks CA; ${ }^{4}$ New York University, New York NY, USA

Background: PsA patients have greater prevalence of cardiovascular disease (CVD), metabolic syndrome (MetS), and cancer than patients without PsA.

Objectives: To examine patient characteristics and disease activity by comorbidity profile among PsA patients.

Methods: This analysis included adults with PsA enrolled in the US Corrona PsA spondyloarthritis Registry from March 2013-March 2017 and followed for $\geq 6$ months. Prevalence (at registry entry) and incidence rate (time to new events after registry entry) of CVD, MetS, and cancer were determined. Patient characteristics and disease activity were described by prevalent comorbidity, with t-tests for means despite skewed data and chi-squared tests for percentages.

Results: The analysis included 1493 patients and 3564 patient-years of followup. Incidences (95\% confidence interval) per 1000 patient-years were $9.4(6.5-$ 13.5), 1.0 (0.3-3.1), and 11.4 (8.2-15.8) for CVD, MetS, and cancer (6.8 [4.510.2] nonmelanoma skin cancer), respectively. PsA patients with (vs without) prevalent CVD, MetS, or cancer were older, and fewer had full-time jobs or private insurance. Patients with (vs without) CVD had higher swollen joint count and mean body surface area, and tended to have higher rates of obesity. Patients with (vs without) MetS tended to have greater disease activity. Patients with (vs without) comorbidities reported less disease activity on patient global assessment.

Data are mean or\%

${ }^{a}>3$ of 4 conditions: hypertension, hyperlipidemia, diabetes mellitus, or obesity

${ }^{\mathrm{b}} P$ value across all categories for employment or body mass index

Conclusions: PsA patients with (vs without) CVD had greater disease activity and those with (vs without) MetS tended to have greater disease activity by physician-derived measures, but PsA patients with (vs without) CVD or MetS reported lower global assessment of disease activity. Patient perception of PsA may mask the effect of comorbid CVD or MetS on disease activity.

Acknowledgements: Amgen Inc. supported this work. Corrona has been supported by AbbVie, Amgen, AstraZeneca, Boehringer Ingelheim, BMS, Crescendo, Eli Lilly and Company, Genentech, GSK, Horizon Pharma USA, Janssen, Momenta Pharmaceuticals, Novartis, Pfizer, Roche, and UCB. Jonathan Latham (PharmaScribe) and Linda Rice (Amgen) provided medical writing support.

Disclosure of Interest: P. Mease Grant/research support from: Abbvie, Amgen, Bristol Myers Squibb, Janssen, Lilly, Novartis, Pfizer, Sun, UCB, Consultant for: Abbvie, Amgen, Bristol Myers Squibb, Janssen, Lilly, Merck, Novartis, Pfizer, Sun, UCB, Zynerba, Speakers bureau: Abbvie, Amgen, Bristol Myers Squibb, Genentech, Janssen, Novartis, Pfizer, UCB, H. Litman Employee of: Corrona, LLC, N. Accortt Shareholder of: Amgen Inc., Employee of: Amgen Inc., S. Rebello Employee of: Corrona, LLC, J. Greenberg Shareholder of: Corrona, LLC, Consultant for: Genentech, Janssen, Pfizer, Eli Lilly, Novartis, Employee of: Corrona, LLC, H. Feng Employee of: Corrona, LLC, M. Gharaibeh Shareholder of: Amgen Inc., Employee of: Amgen Inc., G. Aras Shareholder of: Amgen Inc., Employee of: Amgen Inc., D. Collier Shareholder of: Amgen Inc., Employee of: Amgen Inc. DOI: 10.1136/annrheumdis-2018-eular.1403 